\title{
Objective refraction from aberrometry: theory
}

\author{
Rafael Navarro \\ Instituto de Ciencia de Materiales de Aragón (ICMA) \\ Consejo Superior de Investigaciones \\ Científicas-Universidad de Zaragoza, \\ Facultad de Ciencias \\ Plaza San Francisco s/n \\ 50009 Zaragoza, Spain \\ E-mail: rafaelnb@unizar.es
}

\begin{abstract}
A theoretical framework to formulate and solve the problem of obtaining the objective refraction of an eye from aberrometric data is presented. Matrix formalism was applied to represent lens power and beam vergences in standard clinical, sphere+cylinder $(S+C)$ refraction, and to describe the vergence error of a general aberrated skew ray. The vergence error matrix of each ray passing through the pupil is obtained, and the global refractive error is obtained by simple pupil average. The $2 \times 2$ vergence error matrix of a skew ray can be decomposed into the sum of two even-symmetric and odd-symmetric contributions. The even symmetric part corresponds to classic $S+C$ refractive errors. The odd component can not be corrected with standard lenses. All odd components have zero mean over pupil, and do not contribute to the global refractive error, which is completely determined by $S+C$ components. The contributions of wavefront Zernike modes to the global vergence error were obtained: The contributions of odd orders are zero, but all even HOA, but spherical aberration, contribute to refractive error. The matrix formulation of power and vergence errors provided a direct, simple way to use aberrometers as objective refractometers. ๑ 2009 Society of Photo-Optical Instrumentation Engineers. [DOI: 10.1117/1.3103319]
\end{abstract}

Keywords: aberrometry; refractive error; refraction from aberrations; vergence error objective refraction; refraction from wavefront.

Paper 08381RR received Oct. 24, 2008; revised manuscript received Jan. 23, 2009; accepted for publication Jan. 23, 2009; published online Mar. 25, 2009.

\section{Introduction}

Aberrometry is becoming a common tool in clinical practice. It provides much richer information than other objective techniques to assess refractive errors of the eye (autorrefractometers, retinoscopy, etc.). However, the problem of obtaining the objective refraction from the wave aberration has been elusive and remains unsolved. ${ }^{1}$ Different methods have been proposed to correlate wavefront errors with subjective refraction. ${ }^{2,3}$ Some objective image quality metrics showed a good correlation with subjective visual acuity. ${ }^{4}$ Good visual acuity predictions can also be computed directly through schematic models of early visual processing. ${ }^{5}$ The main problem is that instead of a direct measure of objective refraction, these methods use some image quality metrics (or visual quality for subjective refraction) computed from the wave aberration, so that the predicted refraction is that providing optimal quality under the chosen metrics. Such predictions can never be as accurate as real assessments (subjective or objective) of refractive errors. In fact, many different optical, image and visual quality metrics have been proposed, while different metrics may predict different refractive errors.

The departure hypothesis of this work is that to deal with refractive errors, the direct and possibly right strategy is to strictly focus on refractive errors and correcting lens powers (that is the field of clinical refraction), rather than indirect

Address all correspondence to Rafael Navarro Belsue, ICMA, Consejo Superior de Investigaciones Cientificas-Universidad de Zaragoza, Facultad de CienciasPedro Cerbuna, 12 Zaragoza, Zaragoza 50009 Spain; Tel: 34976762782; Fax: 34976761233;E-mail:rafaelnb@unizares ways such as image quality metrics or wavefront errors. Some early stages toward a direct computation of refractive error prescription from aberrometry have been done already. Power vectors $\left(\mathbf{M}, \mathbf{J}_{0}, \mathbf{J}_{45}\right)$, introduced by Thibos et al., ${ }^{6}$ are useful to obtain the objective refraction from the wave aberration in the presence of second-order aberrations (defocus and astigmatism) only. Unfortunately, in the presence of higher order aberrations (HOA), such a direct relationship no longer applies. Iskander et al. $^{7}$ have proposed the first (to my knowledge) method of objective refraction from monochromatic wavefront aberrations via Zernike power polynomials. Because these power polynomials are not orthogonal, more recent work suggests that the well-known Zernike polynomial basis can be better for analyzing refractive power maps derived from measurements of a radial wavefront slope. ${ }^{8}$ These recent approaches try to solve the problem in a direct way, instead of that based on image quality metrics (indirect method), but they always depart from the wavefront. An essential fact, not considered by any of these direct or indirect approaches, is that wavefront error may not be relevant for objective refraction. Clinical refraction deals with lens powers and beam vergences, following simple additive rules. Wavefronts belong to the field of wave optics, and these two domains might be treated independently. Going back to the origins of aberrometry, it turns out that most early studies of spherical, ${ }^{9,10}$ or chromatic, ${ }^{11,12}$ aberration, where based on measuring the longitudinal shift of focus (vergence error) in diopters for different pupil radius [longitudinal spherical aberration (LSA)] or

$1083-3668 / 2009 / 14(2) / 024021 / 11 / \$ 25.00$ @ 2009 SPIE 
wavelength [longitudinal chromatic aberration (LCA)] respectively, using standard, subjective or objective refraction methods. In other words, standard refraction is directly linked to longitudinal aberration (LA). The main difference between early and modern aberrometry ${ }^{13-15}$ is that today's aberrometers measure transverse displacements of spots [transverse aberrations (TAs)] instead of longitudinal shifts (LA) and then compute the wavefront error (by some numerical integration).

The main goal of this paper is to show that the problem of obtaining the objective refraction (prescription) from aberrometry may be solved within the domain of geometrical optics, which deals with rays, beams, or ray bundles. Waves and wavefronts belong to the field of wave physics, which might be not so useful to study refractive errors.

To achieve this objective, a theoretical framework with the mathematical formulation of vergence and vergence errors is introduced in Section 2. This formulation starts with a brief overview of the well-known theory of classic refraction, with the fundamental concepts of lens power and beam vergences. Then, the vergence error of a general skew ray is analyzed to find possible analogies to classical refraction. The resulting expressions are then applied to obtain the local (ray) and from that, the global (for the whole ray bundle passing through the pupil) vergence error (refraction) from the raw aberrometric data (coordinates of centroinds of spots). Thus, if one can obtain the refractive error from the raw aberrometric data (lateral displacements of spots), the problem of refraction from wave aberration might be less relevant. Nevertheless, that theoretical framework seems powerful enough to provide the contributions of the different wave Zernike modes to the global vergence error. This provides one solution to that problem, but the direct computation from the raw data is implemented and tested as well.

\section{Vergence Error Matrix}

Let us briefly overview some basic ideas about vergence and refractive power. The importance of vergence in clinical refraction comes from the fundamental equation of lenses, written in terms of power and vergences,

$$
V^{\prime}=P+V \text {. }
$$

This equation states that the working principle of a lens is to modify the vergence of the incident (object) beam $V$. Basically, the lens modifies incident vergence by adding its refractive power to the exit (image) beam. The vergences of both object and image beams are given by $V=n / z$ and $V^{\prime}=n^{\prime} / z^{\prime}$ respectively; the lens power is $P=n^{\prime} / f^{\prime}=1 / z_{0}$, where $n, n^{\prime}$ are object and image refractive indices and $z$ and $z^{\prime}$ are the distances of the object and image to the respective principal planes of the lens; $f^{\prime}$ is the focal length of the lens $\left(z_{0}\right.$ $\left.=f^{\prime} / n^{\prime}\right)$. Figure 1 illustrates the concept of vergence error using a classical diagram comparing vergence errors for myopic (upper panel) and hypermetropic (lower panel) eyes. The target vergence to focus the image on the retina is $V_{\mathrm{R}}$ $=n^{\prime} / z_{\mathrm{R}}$, where $z_{\mathrm{R}}$ is the distance from the (image) principal plane of the eye to the retina. The vergence or refractive error will be the difference

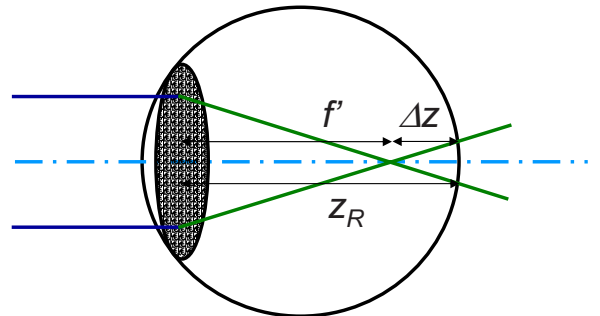

(a)

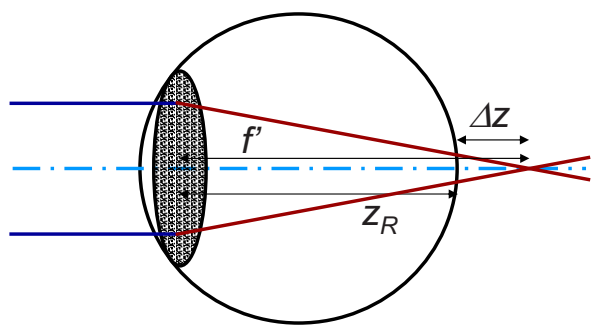

(b)

Fig. 1 Longitudinal defocus $\Delta z$ for (a) myopic and (b) hyperopic eyes. The associated vergence error is $n^{\prime} / \Delta z$.

$$
\Delta V=P_{\mathrm{e}}-V_{\mathrm{R}}=\frac{n^{\prime}}{f^{\prime}}-\frac{n^{\prime}}{z_{\mathrm{R}}}=\frac{n^{\prime}}{z_{\mathrm{R}}+\Delta z}-\frac{n^{\prime}}{z_{R}},
$$

where $\Delta z$ is the longitudinal defocus: $\Delta z<0$ for myopic, $\Delta z>0$ for hypermetropic, and $\Delta z=0$ for emmetropic eyes. From Eqs. (1) and (2) and Fig. 1, one finds two strong reasons to work with powers and vergences rather than axial distances: $(i)$ In the living human eye, one does not have access to the longitudinal defocus $\Delta z$ [Fig. 1, Eq. (2)], and (ii) powers and vergences obey simple additive rules [Eq. (1)]. Clinical refraction basically consists of finding the lens with power $P_{l}=-\Delta V$ by looking for the best (objective or subjective) focus. Therefore, the magnitudes involved in refraction are lens powers, beam vergences, and vergence errors, all given in diopters. Another relevant fact is that the human eye shows the two modes of low-order refractive errors: defocus (sphere) and astigmatism (cylinder). To illustrate the effect of astigmatism we can use the same diagram of Fig. 1, but now let us assume that the upper panel corresponds to a tangential plane $(Z Y)$ and the lower panel to a sagital plane $(Z X)$ of an astigmatic eye. In other words, vergence changes with the meridian, so that we have to rewrite Eq. (1) to take these facts into account

$$
V^{\prime}(\theta)=P_{\mathrm{S}}+P_{\mathrm{C}}^{\theta_{0}}\left(\theta-\theta_{0}\right)+V(\theta),
$$

where $P_{\mathrm{S}}$ is the power of a spherical lens, constant for all meridians, and $P_{\mathrm{C}}^{\theta_{0}}\left(\theta-\theta_{0}\right)=D \cos ^{2}\left(\theta-\theta_{0}\right)=D / 2 \cos 2(\theta$ $\left.-\theta_{0}\right)-D / 2$, where $D$ is the power of the cylindrical lens with $\theta_{0}$ axis. $^{6}$ The expression of the total $(S+C)$ power is that of an ellipse in polar coordinates. This means that (in absence of HOA) a beam affected by defocus and astigmatism will have an elliptical shape with semiaxes $P_{\mathrm{S}}+P_{\mathrm{C}}^{\theta_{0}}$ (for $\theta=\theta_{0}$ ) and $P_{\mathrm{S}}-P_{\mathrm{C}}^{\theta_{0}}$ (for $\theta=\theta_{0}+\pi / 2$ ) respectively. Figure 2 shows that power ellipse as well as the vergence of an ideal beam with its focus on the retina. Obviously, it is not necessary to evaluate 


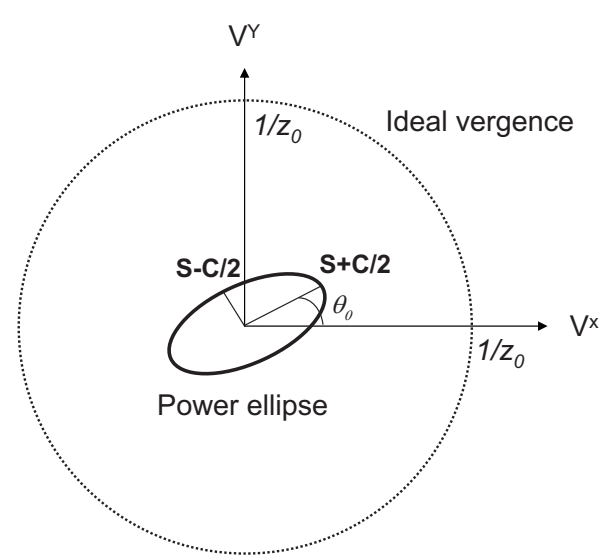

Fig. 2 Diagram of ideal vergence of a spherical beam, and the power ellipse of sphere+cylinder ophthalmic lenses used to compensate the vergence error ellipse of the eye.

vergence for all meridians, because that ellipse is determined by only three parameters: $P_{\mathrm{S}}, P_{\mathrm{C}}, \theta_{0}$. We can represent the elliptical power as a matrix in the Cartesian space. Let us depart from case $\theta_{0}=0$ so that the principal axes of the ellipse are aligned along the horizontal and vertical meridians. Then we have a diagonal power matrix:

$$
\mathbf{P}_{\theta=0}=\left(\begin{array}{cc}
(S+C / 2) & 0 \\
0 & (S-C / 2)
\end{array}\right) .
$$

To pass to a general case, we have to rotate the ellipse, or equivalently, to rotate the system of coordinate. Applying basic linear algebra,

$$
\begin{aligned}
\mathbf{P}_{\theta} & =\mathbf{R}_{\theta} \mathbf{P}_{0} \mathbf{R}_{\theta}^{-1} ; \quad \text { where } \mathbf{R}_{\theta}=\left(\begin{array}{cc}
\cos \theta_{0} & -\sin \theta_{0} \\
\sin \theta_{0} & \cos \theta_{0}
\end{array}\right) \text { and } \mathbf{R}_{\theta}^{-1} \\
& =\mathbf{R}_{\theta}^{T} .
\end{aligned}
$$

After operating, we arrive to

$$
\begin{aligned}
\mathbf{P}_{\theta} & =\left(\begin{array}{cc}
S+C / 2 \cos 2 \theta_{0} & C / 2 \sin 2 \theta_{0} \\
C / 2 \sin 2 \theta_{0} & S-C / 2 \cos 2 \theta_{0}
\end{array}\right) \\
& =\left(\begin{array}{cc}
S+C_{0} & C_{45} \\
C_{45} & S-C_{0}
\end{array}\right) .
\end{aligned}
$$

The elements $\left(S, C_{0}, C_{45}\right)$ have both similarities and differences with the power vectors notation $\left(M, J_{0}, J_{45}\right)$ introduced by Thibos et al.. ${ }^{6}$ The two formulations are quite different because these authors applied Fourier analysis instead of matrix notation. In the absence of HOA, power vectors permit one to solve the problem of refraction from the wavefront. The two notations are equivalent for astigmatism $\left(C_{0}\right.$ $\left.=J_{0}, C_{45}=J_{45}\right)$, but differ because the maximum power $M$ $=S+J$ (with $J^{2}=J_{0}^{2}+J_{45}^{2}$ ) does not appear explicitly under the matrix formalism. Under this formalism, both power and vergence, $\mathbf{P}_{\mathbf{1}}=-\Delta \mathbf{V}$, are matrices.

The advantage of the matrix expressions of power and vergences will become apparent when analyzing aberrometric data. Let us remember that old aberrometric measurements consisted of measuring refractive errors (in diopters) for dif-

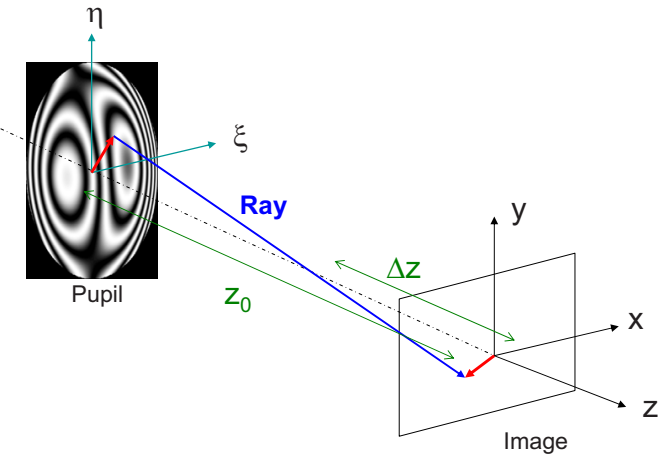

Fig. 3 Trajectory of a general skew ray from the pupil to the image. Skew rays will never cross the axis so that the scalar $\Delta z$ and its associated vergence are not well defined.

ferent pupil radius (LSA), ${ }^{9,10}$ wavelength (LCA), ${ }^{11,12}$ etc. There is a clear link between standard refraction and LA. The problem comes from the fact that modern aberrometers measure transverse displacements of spots, which means two TAs $\left(\mathrm{TA}_{x}, \mathrm{TA}_{y}\right)$ instead of one LA $\left(\mathrm{LA}_{z}\right)$. The following analysis applies directly to ray-tracing aberrometers, but most types of aberrometers, such as the popular Hartmann-Shack (H-S) wavefront sensors are equivalent. ${ }^{16}$ The difference is that in the H-S sensor, $X$ and $Y$ are displacements of spots from their respective aberration-free positions. If $\xi, \eta$ are the (pupil) coordinates of the optical axis of a lenslet, then $X(\xi, \eta)$ $=X^{\prime}(\xi, \eta)-\xi$ and $Y(\xi, \eta)=Y^{\prime}(\xi, \eta)-\eta$, where $X^{\prime}, Y^{\prime}$ are coordinates of the image spot of that lenslet; $Z_{0}$ is the focal length of the lenslet.

When HOA are present, the diagram of Fig. 1 is not valid anymore. Instead, Fig. 3 shows a diagram of a general (aberrated) skew ray. The ray is defined by the straight line connecting one point at the exit pupil, with coordinates $\xi, \eta$, to another point at the image plane (retina), with coordinates $X$, $Y$. Figure 4 shows a front view (projection on the $X Y$ plane) of Fig. 3. The initial origin of coordinates is the pupil center; the $Z$ axis is the line connecting the center of the pupil with the ideal image spot. To compute the vergence error, we can apply Eq. (2) to that particular ray. However, a skew ray may never intersect the optical axis, so that $\Delta z$ may be undefined as well. This problem may be overcome if we first look at the projections of that ray on the $X Z$ and $Y Z$ planes. For each projection, the ray trajectory will resemble one of the rays in Fig. 1 (i.e., the projections of the skew ray do intersect the axis again). In general, we will have a well-defined LA $\Delta z$ for any projection coplanar to the $Z$-axis, but the resulting LA may be different for each projection; $\Delta z_{x} \neq \Delta z_{y}$, in general. Therefore, we have to consider two vergence errors for the two orthogonal projections

$$
\Delta V^{x}=\frac{1}{z_{0}+\Delta z_{x}}-\frac{1}{z_{0}} ; \quad \Delta V^{y}=\frac{1}{z_{0}+\Delta z_{y}}-\frac{1}{z_{0}} .
$$

Note two small changes in notation with respect to Eq. (2). Now $z$ distances are optical distances because they include normalization by the refractive index. The second change is implicit and accounts for the fact that, in the human eye, the target vergence is given by the optical distance to the retina, 
so that $z_{0}=z_{\mathrm{R}}$, whereas the focal length $f^{\prime}$ is irrelevant and possibly unknown. From Fig. 2, we can establish the equality of right and left tangents of the angle formed by the ray and the $Z$-axis

$$
-\frac{X}{\Delta z_{x}}=\frac{\xi}{z_{0}-\Delta z_{x}} ; \quad-\frac{Y}{\Delta z_{y}}=\frac{\eta}{z_{0}-\Delta z_{y}}
$$

We can solve both equations for $\Delta z$ and substitute in Eq. (4) to obtain

$$
\Delta V_{\xi}^{x}=\frac{X}{z_{0} \xi} ; \quad \Delta V_{\eta}^{y}=\frac{Y}{z_{0} \eta} .
$$

These equations state that the vergence error for a particular ray is equal to the ideal vergence times the ratio between pupil and image coordinates of the ray.

Obviously, the above analysis is incomplete because the three-dimensional (3-D) problem was reduced to two twodimensional projections, whereas the 3-D geometry cannot be recovered simply by two orthogonal projections as suggested by Fig. 4. In fact, for these skew rays there is no rotation around the $Z$-axis to make it coplanar to the $Z$-axis. The cross vergences are nonzero, in general, so that the vergence error of a skew ray is a $2 \times 2$ matrix

$$
\Delta \mathbf{V}=\frac{1}{z_{0}}\left(\begin{array}{cc}
\frac{X}{\xi} & \frac{X}{\eta} \\
\frac{Y}{\xi} & \frac{Y}{\eta}
\end{array}\right) .
$$

This result implies bad news: This matrix is not symmetric (four parameters instead of three), and its determinant $\operatorname{det}(\Delta \mathbf{V})=|\Delta \mathbf{V}|=0$, so that it is not diagonalizable. Therefore, the vergence error of a skew ray cannot be corrected in a standard way $(S+C)$. However, we can decompose such illposed matrix by two contributions,

$$
\begin{aligned}
\Delta \mathbf{V} & =\frac{1}{z_{0}}\left[\begin{array}{cc}
\frac{X}{\xi} & \frac{1}{2}\left(\frac{X}{\eta}+\frac{Y}{\xi}\right) \\
\frac{1}{2}\left(\frac{X}{\eta}+\frac{Y}{\xi}\right) & \frac{Y}{\eta}
\end{array}\right] \\
& +\frac{1}{z_{0}}\left[\begin{array}{cc}
0 & \frac{1}{2}\left(\frac{X}{\eta}-\frac{Y}{\xi}\right) \\
-\frac{1}{2}\left(\frac{X}{\eta}-\frac{Y}{\xi}\right) & 0
\end{array}\right]=\mathbf{V}+\mathbf{V}_{\mathbf{c}} .
\end{aligned}
$$

The first even symmetric matrix $\mathbf{V}$ does represent an ellipsoid. We know that an elliptical vergence error can be corrected by combining spherical and cylindrical lenses. The oddsymmetric matrix corresponds to the odd-symmetric "comatic" vergence error. To correct $\mathbf{V}_{\mathbf{c}}$, one could construct an element consisting of two half-cylinders, one-half with positive and the other half with negative power, and place it with its axis at $45 \mathrm{deg}$. However, such an optical element is not conventional; thus, its correction is outside the scope of conventional clinical refraction.

Now, if we focus on matrix $\mathbf{V}$, its elements are well defined for all rays, except for those coming from the pupil axes

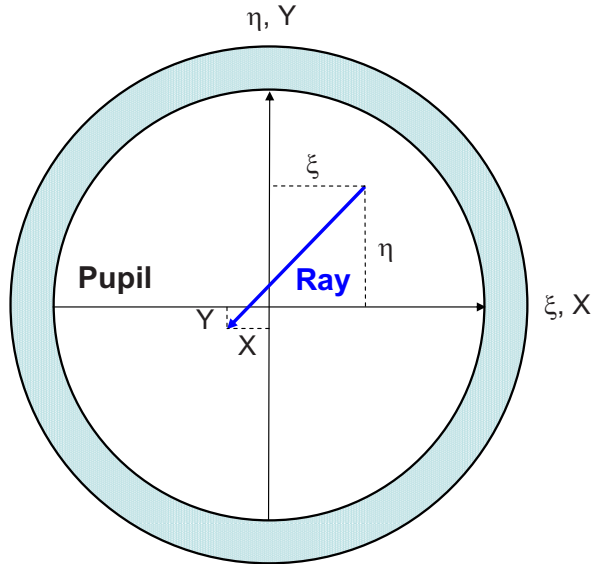

Fig. 4 Front view (projection on the $X Y$ plane) of a skew ray similar to that of Fig. 3. There are four possible components of the vergence error because each transverse aberration depends on both pupil coordinates.

$\xi=0$ or $\eta=0$, where the left or right columns of the matrix show singularities, respectively. If we call $\mathbf{E}=z_{0} \mathbf{V}$, this matrix contains ratios of pupil and image coordinates. It can be understood as an affine matrix operator, which transforms the ideal spherical beam (two equal semiaxes: $1 / z_{0}$ ) into an elliptical one. In other words, we can define an elliptical beam associated to each skew ray. Thus, we can apply the refractive correction $\mathbf{P}_{\mathbf{1}}=-\mathbf{V}$ (the actual physical beam will have also a cross-comatic component, but that is not treatable with standard spheres and cylinders). Using the matrix expression [Eq. (6)] to each ray passing through the pupil, we obtain

$$
-\frac{\mathbf{E}(\xi, \eta)}{z_{0}}=\left[\begin{array}{cc}
S(\xi, \eta)+C_{0}(\xi, \eta) & C_{45}(\xi, \eta) \\
C_{45}(\xi, \eta) & S(\xi, \eta)-C_{0}(\xi, \eta)
\end{array}\right] \text { diopters. }
$$

This expression permits one to apply a classical refractive correction on [Eqs. (3) and (6) in matrix form] to each ray of the bundle passing through the pupil. This correction requires three elements, a spherical lens with power $S$, a horizontal cylinder $C_{0}$, plus a diagonal cylinder $C_{45}$ (this correction can be also achieved by two lenses $S+C$ or by a single toric element). The complete correction for the ray requires an additional odd-symmetric element $H \pm C_{45}$ (half-positive, halfnegative cylinder) as the one shown in Fig. 5, aligned along the diagonal to correct "comatic" vergence. The upper panel shows a perspective view of this type of odd-symmetric lens, with half-negative and half-positive cylinders. The lower panel illustrates the effect of that element, which is to produces a half-diverging (negative cylinder part) and a halfconverging (positive cylinder part) beam.

Under this formulation, aberrometry may be understood as standard refraction, with the difference that such refraction is particularized to each ray (or sample) $(\xi, \eta)$ of the entire bundle passing through the pupil. The conclusion is that one can perform a microrefraction to obtain the corresponding microlens prescription. However, vergence error is a $2 \times 2$ matrix, thus requiring four elements for its complete characterization, and potential compensation. The main difference with standard refraction is the presence of odd-symmetric diagonal 


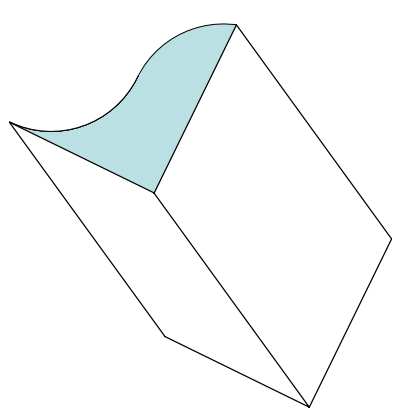

(a)

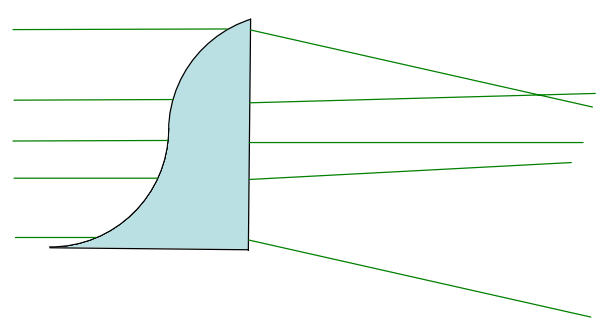

(b)

Fig. 5 The odd-symmetric lens consisting of two half-positive and half-negative cylinders. (a) a perspective view. (b) The effect of that lens is to produce a half-diverging and a half-converging beam. This type of lens could correct the cross-comatic vergence error when aligned along the 45-deg diagonal.

elements (i.e., comatic vergence errors). If we leave that odd term (which may have similarities with irregular astigmatism in clinics) apart, then we can apply the theory and practice of refraction to correct the $S+C$ vergence error of any skew ray in an aberrated beam. One may obtain a complete correction, by canceling all vergence errors, so that the ray will impact the image at the ideal image point; but this requires introducing a fourth element: $H \pm C_{45}$. This means aberration correction via standard refraction, might be possible for each ray, which may have implications in alternative implementations of adaptive optics. Nevertheless, there is a potential problem for rays passing through the pupil axes $(\xi, 0)$ or $(0, \eta)$, where vergence errors could present singularities $(X / 0$ or $Y / 0)$. To avoid these singularities, it is important to cancel prismatic effects by choosing the chief ray as the $Z$-axis, so that $X(0,0)=Y(0,0)=0$, as suggested by ANSI standards. However, this is a necessary but not a sufficient condition for totally avoiding singularities along pupil axes in general. The issues of how to deal with these singularities, or how to treat vergence errors associated to prismatic effects, are open problems that may deserve further study. On the other hand, when the vergence error is constant for all rays (i.e., when we have pure second-order aberrations, as shown in Section 3), all correcting "microlenses" will be equal and we can use a single lens for the whole pupil. In the presence of HOA, the problem is to find the best single toric lens to obtain an optimal balance for the whole beam.

\section{Vergence Error from Aberrometric Data}

The above results suggest a direct way to compute the vergence error $\mathbf{V}$ for each ray (sample), from the aberrometric centroids $X(\xi, \eta), Y(\xi, \eta)$ and their corresponding pupil coor-

\section{Standard Scheme}

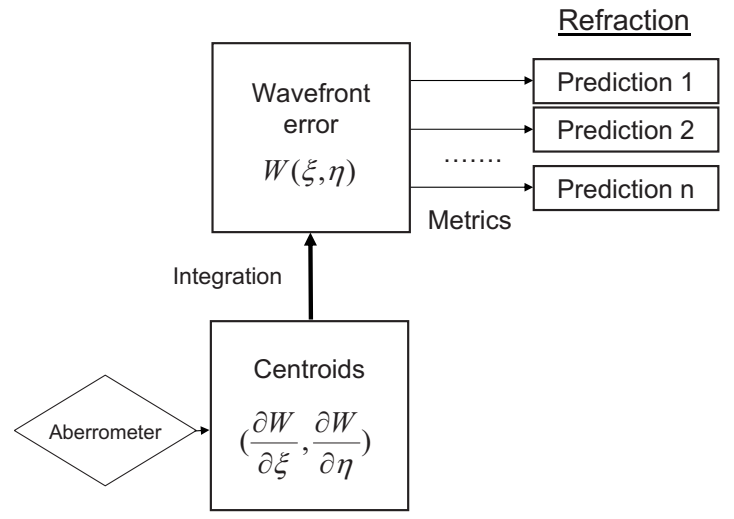

(a)

\section{Proposed Scheme}

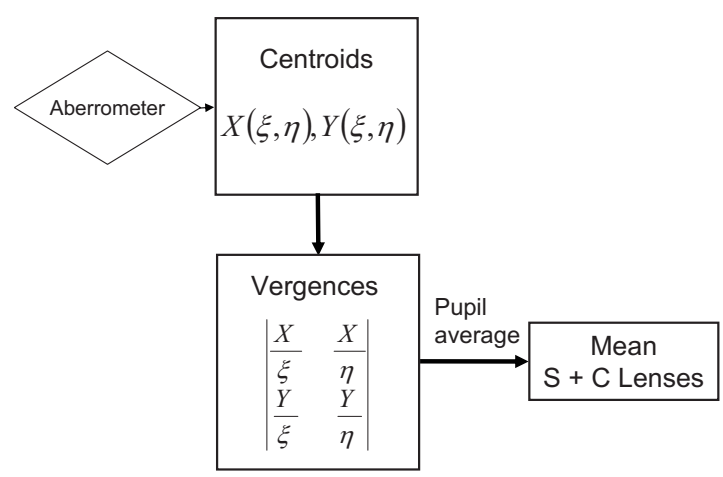

(b)

Fig. 6 Diagram comparing (a) standard and (b) proposed methods to assess the objective refraction with an aberrometer.

dinates $(\xi, \eta)$. These coordinates $X(\xi, \eta), Y(\xi, \eta)$ are the direct result of aberrometric measurements (raw data) and are typically used to plot the classic spot diagrams. This strategy is totally opposite to that attempted in most studies until now. As shown in the upper panel of Fig. 6, the standard scheme used in the great majority of studies consists of reconstructing the wavefront by numerical integration first. The direct use of the second-order Zernike terms is known to give a wrong prescription in the presence of HOA. ${ }^{2}$ Different indirect strategies have been proposed based on the optimization of some image or visual quality metrics. However, the theoretical framework presented in Section 2 suggests that the problem of refraction from aberrometry may have little to do with wave optics. On the contrary, vergences and refraction belong to the domain of geometrical optics (rays). Thus, wavefront reconstruction might be an unnecessary stage for the goal of clinical refraction. The proposed scheme (lower panel in Fig. 6 ) is a direct computation of the vergence error matrix, which provides the objective local refraction for each pupil sample (ray, lenslet, etc.) Once we have the local refraction across the pupil, there are different possibilities to pass from that local set of vergences to the global refractive error. The mean, or simple average (or pupil integral), is the best estimator in a 
least-squares sense, whereas the mode might be a better estimator under robust nonlinear criteria. Of course, when the distribution is symmetric (no skewness), both estimators are equal. For that reason, the most natural, simplest choice is to compute the mean vergence over the (desired) pupil.

To compute the pupil average, one has to solve the problem of the potential singularities, $(X / 0, Y / 0)$ occurring along the $\xi, \eta$ pupil axes. For instance, prismatic effects will give infinity vergence error. ANSI standards to report wave aberration data recommend choosing the chief ray as the $Z$-axis. This choice removes all prismatic contributions and guarantees that $X(0,0)=Y(0,0)=0$, possibly making that singularity avoidable at the origin. However, this choice does not totally guarantee possible singularities along the axes, where $\xi=0$ or $\eta=0$. As we will see below, positive $(+\infty)$ and negative $(+\infty)$ singularities cancel each other by pupil averaging. However, in real practice, we have a limited number of samples, so that the contributions of these singular points might bias the result in a sampling-dependent way. These two issues of singularities and sampling will be further analyzed in Section 4.

Consequently, because those data points along the pupil axes might present singularities (infinity or very high values), the safer strategy is to leave singularities away when computing the global refractive error from a limited number of samples. The estimation of the average vergence over the pupil will be

$$
\langle\mathbf{V}\rangle=\frac{1}{N z_{0}}\left[\begin{array}{cc}
\sum_{i, j ; \xi \neq 0} \frac{X_{i, j}}{\xi_{i, j}} & 1 / 2\left(\sum_{i, j ; \eta \neq 0} \frac{X_{i, j}}{\eta_{i, j}}+\sum_{i, j ; \xi \neq 0} \frac{Y_{i, j}}{\xi_{i, j}}\right) \\
1 / 2\left(\sum_{i, j ; \eta \neq 0} \frac{X_{i, j}}{\eta_{i, j}}+\sum_{i, j ; \xi \neq 0} \frac{Y_{i, j}}{\xi_{i, j}}\right) & \sum_{i, j ; \eta \neq 0} \frac{Y_{i, j}}{\eta_{i, j}}
\end{array}\right],
$$

where $N$ is the number of spots used to compute the average. Note that the potentially singular points are not included for convenience.

It is worth remarking that the computation of this average may not require Zernike polynomials, or equivalent expansion, because it can be directly computed from the raw aberrometric data (centroids). Thus far, the whole formulation has been developed without mention of wavefronts or wave aberrations. In what follows, we analyze the link between geometric and wave optics and analyze possible implementations.

\section{Contributions of Wave Zernike Modes}

Let us start with the analysis of the trivial case with pure second-order aberrations to verify that we obtain consistent results. The contribution of each Zernike wavefront error mode to the vergence error will be analyzed next.

\subsection{Pure Second-Order Wavefront Errors}

To illustrate these ideas, let us consider a HOA-free wavefront, with only second-order Zernike defocus and astigmatism modes, following the ANSI standard,

$$
w=c_{2}^{-2} Z_{2}^{2}+c_{2}^{0} Z_{2}^{0}+c_{2}^{2} Z_{2}^{2} .
$$

To connect wavefront error to vergence error, let us remember the fundamental equations of aberrometry, linking wave and ray optics ${ }^{17}$

$$
X=\frac{z_{0} \partial W}{\partial \xi} ; \quad Y=\frac{z_{0} \partial W}{\partial \eta} .
$$

Using these expressions, we can now compute the average vergence $\langle\mathbf{V}\rangle$ integrating over the pupil. When using Zernike polynomials, pupil coordinates are normalized to the pupil radius, so that $\xi=R \bar{\xi}$ and $\eta=R \bar{\eta}$,

$$
\begin{aligned}
\left\langle V_{\bar{\xi}}^{x}\right\rangle & =\left\langle\frac{1}{R \bar{\xi}}\left(c_{2}^{-2} \frac{\partial Z_{2}^{2}}{R \partial \bar{\xi}}+c_{2}^{0} \frac{\partial Z_{2}^{0}}{R \partial \bar{\xi}}+c_{2}^{-2} \frac{\partial Z_{2}^{2}}{R \partial \bar{\xi}}\right)\right\rangle \\
& =\frac{1}{R^{2}}\left(2 \sqrt{6} c_{2}^{-2}\left\langle\frac{\bar{\eta}}{\bar{\xi}}\right\rangle+4 \sqrt{3} c_{2}^{0}+2 \sqrt{6} c_{2}^{2}\right)=\frac{4 \sqrt{3} c_{2}^{0}}{R^{2}}+\frac{2 \sqrt{6} c_{2}^{2}}{R^{2}}
\end{aligned}
$$

because the average over a circle of unit radius $\langle\bar{\eta} / \bar{\xi}\rangle_{\text {circ }}$ $=(1 / 2) \int_{-\pi}^{+\pi} \operatorname{tg} \theta d \theta=0$. We can apply the same procedure to obtain the other elements of the vergence matrix

$$
\left\langle V_{\eta}^{y}\right\rangle=\frac{4 \sqrt{3} c_{2}^{0}}{R^{2}}-\frac{2 \sqrt{6} c_{2}^{2}}{R^{2}} ; \quad\left\langle V_{\eta}^{x}\right\rangle=\left\langle V_{\bar{\xi}}^{y}\right\rangle=\frac{2 \sqrt{6} c_{2}^{-2}}{R^{2}} .
$$

When coefficients $c_{n}^{m}$ are given in micrometers $\left(10^{-6} \mathrm{~m}\right)$, and $R^{2}$ in millimeters squared $\left(10^{-6} \mathrm{~m}^{2}\right)$, it turns out that the refractive errors are already given in diopters

$$
\begin{aligned}
\langle\mathbf{V}\rangle & =\left(\begin{array}{cc}
\frac{4 \sqrt{3} c_{2}^{0}}{R^{2}}+\frac{2 \sqrt{6} c_{2}^{2}}{R^{2}} & \frac{2 \sqrt{6} c_{2}^{-2}}{R^{2}} \\
\frac{2 \sqrt{6} c_{2}^{-2}}{R^{2}} & \frac{4 \sqrt{3} c_{2}^{0}}{R^{2}}-\frac{2 \sqrt{6} c_{2}^{2}}{R^{2}}
\end{array}\right) \\
& =\left(\begin{array}{cc}
S+J_{0} & J_{45} \\
J_{45} & S-J_{0}
\end{array}\right) .
\end{aligned}
$$

This is a well-known result: In the absence of HOA, the refractive error is as expected from the second-order wave aberration coefficients. However, the results are different in the presence of HOA. 


\subsection{Refraction in the Presence of Arbitrary HOA}

To compute the effect of HOA to the average vergence error, we can expand the contribution of each individual Zernike mode as follows:

$$
\left\langle V_{\xi}^{x}\right\rangle_{n}^{m}=\left\langle\frac{\partial Z_{n}^{m}}{\xi \partial \xi}\right\rangle=\nu_{0}+\sum_{n^{\prime}, m^{\prime}}^{n-2, m-2} \nu_{n^{\prime}}^{m^{\prime}}\left\langle Z_{n^{\prime}}^{m^{\prime}}\right\rangle+\sum_{0}^{n-2} \nu_{-1}^{p}\left\langle\frac{\eta^{p}}{\xi}\right\rangle=\nu_{0}
$$

A well-known property of Zernike polynomials is $\left\langle Z_{n^{\prime}}^{m^{\prime}}\right\rangle=0$, except for piston: $\left\langle Z_{0}^{0}\right\rangle=1$ (nonzero Zernike modes contribute to the variance but not to the average). In addition, the center symmetry of pupil coordinates also cancels the average of coordinate ratios of the form $\left\langle\eta^{p} / \xi\right\rangle=0$. For the demonstration, we consider already normalized pupil coordinates. Then we have the following expressions, which show that all the averages are zero up to third order:

$$
\left.\left\langle\frac{1}{\xi}\right\rangle=\left\langle\frac{1}{\cos \theta}\right\rangle=\int_{-\pi}^{+\pi} \frac{1}{\cos \theta} d \theta=\ln \left|\tan \left(\frac{\theta}{2}+\frac{\pi}{4}\right)\right|\right]_{-\pi}^{+\pi}=0
$$

$$
\left.\left\langle\frac{\eta}{\xi}\right\rangle=\left\langle\frac{\sin \theta}{\cos \theta}\right\rangle=\int_{-\pi}^{+\pi} \operatorname{tg} \theta d \theta=-\ln |\cos \theta|\right]_{-\pi}^{+\pi}=0
$$

$$
\begin{aligned}
\left\langle\frac{\eta^{2}}{\xi}\right\rangle & =\left\langle\frac{\sin ^{2} \theta}{\cos \theta}\right\rangle=\int_{-\pi}^{+\pi} \sin \theta \operatorname{tg} \theta d \theta \\
& =(\ln |\sec \theta+\tan \theta|-\sin \theta)]_{-\pi}^{+\pi}=0 .
\end{aligned}
$$

Similar demonstrations are obtained for the inverse expressions $\left\langle\xi^{p} / \eta\right\rangle=0$ (one may simply rotate $\pi / 2$ the integration limits $[-\pi / 2$ to $3 \pi / 2]$ to arrive to the same result). Note that higher $p$ orders will keep the same symmetry so that pupil average will always be zero. Therefore, we can ignore these terms with negative orders $\left(\xi^{-1}, \eta^{-1}\right)$ in Eq. (19) (and similarly for the other elements of $V$ ). Therefore, we only need to compute the piston term of the Zernike polynomial expansion obtained for each contribution. In particular, it is straightforward to show that the contribution (piston term) of third-order Zernike modes to the average vergence error is zero (this is also true for fifth and higher odd orders, in general),

$$
\begin{aligned}
\left\langle V_{\xi}^{x}\right\rangle_{3}^{m}= & \left\langle\frac{\partial Z_{3}^{m}}{\xi \partial \xi}\right\rangle=+\nu_{1}^{-1}\left\langle Z_{1}^{-1}\right\rangle+\nu_{1}^{1}\left\langle Z_{1}^{1}\right\rangle+\nu_{-1}^{0}\left\langle\frac{1}{\xi}\right\rangle+\nu_{-1}^{1}\left\langle\frac{\eta}{\xi}\right\rangle \\
& +\nu_{-1}^{2}\left\langle\frac{\eta^{2}}{\xi}\right\rangle=0 .
\end{aligned}
$$

The corresponding expression for fourth-order terms can be simplified somewhat to only piston and second-order terms,

$$
\begin{aligned}
\left\langle V_{\xi}^{x}\right\rangle_{4}^{m}= & \left\langle\frac{\partial Z_{4}^{m}}{\xi \partial \xi}\right\rangle=\nu_{0}+\nu_{2}^{-2}\left\langle Z_{2}^{-2}\right\rangle+\nu_{2}^{0}\left\langle Z_{2}^{0}\right\rangle+\nu_{2}^{-2}\left\langle Z_{2}^{2}\right\rangle \\
& +\nu_{-1}^{0}\left\langle\frac{1}{\xi}\right\rangle+\nu_{-1}^{1}\left\langle\frac{\eta}{\xi}\right\rangle+\nu_{-1}^{2}\left\langle\frac{\eta^{2}}{\xi}\right\rangle=\nu_{0} .
\end{aligned}
$$

By using these (and equivalent expressions for the other elements of the vergence matrix), it is possible to compute the exact contributions of high-order aberrations to the average vergence error. The results for the different wavefront Zernike modes are summarized in Table 1 up to fifth order. Sixth-order spherical aberration is also included because it might take significant values in normal eyes. From Table 1, it is patent that even order HOA do contribute to the average vergence error, whereas the contributions of odd aberrations are canceled by averaging (integration) over the circular pupil. Fourth-order astigmatism, tetrafoil, and sixth-order spherical aberration appear to contribute to the vergence error. Interestingly, there is no contribution from spherical aberration $Z_{4}^{0}$. If we particularize Eq. (22) for $Z_{4}^{0}$ (in Cartesian coordinates), then

$$
\left\langle\frac{\partial Z_{4}^{0}}{\xi \partial \xi}\right\rangle=\left\langle 12 \sqrt{5}\left(2 \xi^{2}+2 \eta^{2}-1\right)\right\rangle=12 \sqrt{\frac{5}{3}}\left\langle Z_{2}^{0}\right\rangle=0,
$$

and equally for $\eta$ and cross vergences, due to the revolution symmetry of $Z_{4}^{0}$. It is interesting to note that the fourth-order spherical aberration translates into a pure "defocus" $Z_{2}^{0}$ vergence mode. In other words, the fourth-order spherical aberration does not contribute to vergence error because the pupil average of any Zernike polynomial is zero. A possible explanation for the zero contribution is that the $Z_{4}^{0}$ polynomial includes a defocus term, which already discounts such a contribution (as a consequence of orthogonality between $Z_{2}^{0}$ and $Z_{4}^{0}$ ). However, the sixth-order spherical aberration $Z_{6}^{0}$ contributes again. Another interesting case is $Z_{4}^{-4}$ (diagonal tetrafoil) having odd-symmetric cross vergences $\left(\Delta V_{\eta}^{x}=-\Delta V_{\xi}^{y}\right)$ so that its contribution to the even vergence error is canceled. $Z_{4}^{-4}$ is the only even mode (up to fourth order) with a pure contribution to the comatic vergence error $\mathbf{V}_{\mathbf{c}}$. On the other hand, terms with $m \geqslant 0$ (even angular) only contribute to direct vergences (diagonal elements), whereas terms with $m<0$ (odd angular) only contribute to the even cross vergences. The rest of the sixth-order contributions are not included in Table 1 because these aberrations are quite small in normal eyes; thus, their contributions are expected to be small. Nonetheless, their computation is straightforward, using the method described above.

In summary, Table 1 represents the solution to pass from wavefront aberration modes to average vergence errors under the present matrix formulation of the problem. These are direct conversions from micrometers of wave root-mean-square error to diopters of vergence, when pupil radius $R$ is in millimeters. An important aspect is that such conversion involves passing from a scalar magnitude to a $2 \times 2$ matrix operator (representing the action of lenses). 
Table 1 Contributions of wave Zernike modes $Z_{n}^{m}$ to the (pupil) average vergence error. When the Zernike coefficients $C_{n}^{m}$ are in micrometers and pupil radius $R$ in millimeters, then the vergences are in

diopters.

\begin{tabular}{|c|c|c|c|c|c|}
\hline$n$ & $m$ & $\Delta V_{\xi}^{x}$ & $\Delta V_{\eta}^{y}$ & $\Delta V_{\eta}^{x}$ & $\Delta V_{\xi}^{y}$ \\
\hline 2 & -2 & 0 & 0 & $\frac{2 \sqrt{6} c_{2}^{2}}{R^{2}}$ & $\frac{2 \sqrt{6} c_{2}^{2}}{R^{2}}$ \\
\hline 2 & 0 & $\frac{4 \sqrt{3} c_{2}^{2}}{R^{2}}$ & $\frac{4 \sqrt{3} c_{2}^{2}}{R^{2}}$ & 0 & 0 \\
\hline 2 & 2 & $\frac{2 \sqrt{6} c_{2}^{2}}{R^{2}}$ & $-\frac{2 \sqrt{6} c_{2}^{2}}{R^{2}}$ & 0 & 0 \\
\hline 3 & All & 0 & 0 & 0 & 0 \\
\hline 4 & -4 & 0 & 0 & $\frac{2 \sqrt{10} c_{4}^{-4}}{R^{2}}$ & $-\frac{2 \sqrt{10} c_{4}^{-4}}{R^{2}}$ \\
\hline 4 & -2 & & & $\frac{2 \sqrt{10} c_{4}^{-2}}{R^{2}}$ & $\frac{2 \sqrt{10} c_{4}^{-2}}{R^{2}}$ \\
\hline 4 & 0 & 0 & 0 & 0 & 0 \\
\hline 4 & 2 & $-\frac{2 \sqrt{10} c_{4}^{2}}{R^{2}}$ & $\frac{2 \sqrt{10} c_{4}^{2}}{R^{2}}$ & 0 & 0 \\
\hline 4 & 4 & $-\frac{2 \sqrt{10} c_{4}^{4}}{R^{2}}$ & $-\frac{2 \sqrt{10} c_{4}^{2}}{R^{2}}$ & 0 & 0 \\
\hline 5 & All & 0 & 0 & 0 & 0 \\
\hline 6 & 0 & $\frac{4 \sqrt{7} c_{6}^{0}}{R^{2}}$ & $\frac{4 \sqrt{7} c_{6}^{0}}{R^{2}}$ & 0 & 0 \\
\hline
\end{tabular}

\subsection{Direct Method: Computer Simulations}

Table 1 provides a method to compute the refractive error from the wave aberration. However, the direct method in aberrometry (lower panel in Fig. 6) would be to compute the vergence error matrix from the transverse aberrations (raw data). Different computer simulations were carried out to test this direct method and to analyze the effects of factors such as discrete sampling or noise. Several H-S wavefront sensors with square microlenses, all of them covering a $6-\mathrm{mm}$ pupil diameter but having a different number of microlenses, were simulated to study the effect of sampling. Pure Zernike modes (same magnitude of $1 \mu \mathrm{m}$ in all cases) were generated to obtain their respective contributions to the vergence error. The idea was to obtain, numerically, the same contributions as in Table 1 for different numbers of samples. Noise was not introduced in this case to analyze the effect of sampling. The results are given in Table 2 for the different numbers of samples considered. These total numbers of samples (microlenses) correspond to $11,21,101$, and 501 samples along one dimension (axis), respectively. The $\infty$ column corresponds to the direct application of the expressions of Table 1. The result is consistent again for the contributions by second-order aberration modes, $n=2$ (pure refractive errors), which are unaffected by sampling. In addition, all expected zero contributions are unbiased (numerical results were $<10^{-16}$ ), except for the fourth-order spherical aberration, which shows a significant error. The absolute value of the error increases when the number of samples decreases. The rest of fourth-order modes shows a similar behavior, with a tendency to progressively underestimate the contributions to the vergence error as the number of samples decreases. For $n=6$, this sampling bias seems higher. Comparing columns, it seems that a minimum number of samples, of the order of 1000 , seems necessary to guarantee a good accuracy. The reason is twofold: First, as the number of samples decrease, the relative contribution of the samples along the axes, not considered in the average, is higher (compare 11/89 to 501/196,933). Second, the computation of the integral (average) over the circular pupil is less exact. Unfortunately, most aberrometers have a limited number of samples, so that a plain implementation of Eq. (13) could potentially provide biased contributions of HOA. Therefore, practical implementations would require one to include interpolation methods or numerical integration algorithms (i.e., Simpson's rule, basis functions, etc.) to improve accuracy.

The next series of computer simulations consisted of adding different amounts of random noise to the ideal aberrometric data (transverse aberrations $X, Y$ ). Two levels of random noise, 5 and $10 \%$, were simulated in several cases of pure and mixed Zernike modes for the case of H-S sensor with 341 microlenses. The error level in the resulting vergence was lower than the level of noise in the input data in all cases. For a $5 \%$ input noise, typical noise level at the output (vergence) was $\sim 1 \%$; for the case of $10 \%$ noise, the output error was 
Table 2 Effect of the number of samples to the direct numerical computation of the vergence error (in diopters). Column 3 shows those elements of the vergence error matrix different from zero with their signs; columns 4-8 correspond to the results obtained for different numbers of microlenses (samples).

\begin{tabular}{|c|c|c|c|c|c|c|c|}
\hline$n$ & $M$ & $\begin{array}{c}\text { Vergence } \\
\text { elements } \neq 0\end{array}$ & 89 & 341 & 7989 & 196,933 & $\begin{array}{c}\infty \\
\text { (Table 1) }\end{array}$ \\
\hline 2 & -2 & $\Delta V_{\eta}^{x}=\Delta V_{\xi}^{y}=$ & 0.5443 & 0.5443 & 0.5443 & 0.5443 & 0.5443 \\
\hline 2 & 0 & $\Delta V_{\xi}^{x}=\Delta V_{\eta}^{y}=$ & 0.7698 & 0.7698 & 0.7698 & 0.7698 & 0.7698 \\
\hline 2 & 2 & $\Delta V_{\tilde{\xi}}^{x}=-\Delta V_{\eta}^{\gamma}=$ & 0.5443 & 0.5443 & 0.5443 & 0.5443 & 0.5443 \\
\hline 3 & All & & 0 & 0 & 0 & 0 & 0 \\
\hline 4 & -4 & $\Delta V_{\eta}^{x}=-\Delta V_{\xi}^{Y}=$ & 0.5540 & 0.6450 & 0.6917 & 0.7002 & 0.7027 \\
\hline 4 & -2 & $\Delta V_{\eta}^{x}=\Delta V_{\xi}^{y}=$ & 0.5008 & 0.6557 & 0.6946 & 0.6998 & 0.7027 \\
\hline 4 & 0 & $\Delta V_{\xi}^{x}=\Delta V_{\eta}^{\gamma}=$ & -0.0752 & 0.0152 & 0.0041 & -0.00054 & 0 \\
\hline 4 & 2 & $-\Delta V_{\xi}^{x}=\Delta V_{\eta}^{y}=$ & 0.6071 & 0.6342 & 0.6888 & 0.7006 & 0.7027 \\
\hline 4 & 4 & $-\Delta V_{\xi}^{x}=-\Delta V_{\eta}^{\gamma}=$ & 0.5540 & 0.6450 & 0.6917 & -0.7002 & 0.7027 \\
\hline 5 & All & & 0 & 0 & 0 & 0 & 0 \\
\hline 6 & 0 & $\Delta V_{\xi}^{x}=\Delta V_{\eta}^{y}=$ & 0.6353 & 0.9994 & 1.1438 & 1.1668 & 1.1759 \\
\hline
\end{tabular}

$\sim 2 \%$. In summary, random noise does not seem to affect the computation of vergence errors. Integration over the pupil area seems to reduce the potential impact of random noise. However, the accuracy of the direct method appears to depend on the number of aberrometric samples.

\subsection{Examples}

Let us see how to apply the results of Table 1 with a couple of examples. The first example corresponds to aberrometric data of the author's right eye for a 6-mm pupil. The clinical prescription was $S=0$ (because defocus was compensated effectively during aberrometric measurements by a Badal lens system); cylinder $C=-0.5 \mathrm{D}$ at $15 \mathrm{deg}$. The third column of Table 3 shows the Zernike coefficients for this eye (only for contributing even orders) in micrometers, and columns four to six correspond to the contributions of each Zernike mode to the vergence error matrix elements. The total average vergence predicted only from second-order Zernike modes is $S$ $=-0.1 \mathrm{D}, C=-0.45 \mathrm{D}$ at $7.5 \mathrm{deg}$. If we now include the contributions of HOA, the resulting refraction is $S=0.008 \mathrm{D}, C$ $=-0.53 \mathrm{D}$ at $12 \mathrm{deg}$, which is closer to the prescription. For this particular subject, both prescription and aberrations are small; thus, the changes are not very spectacular. In a postLASIK eye with higher levels of HOA, the prescription predicted by only the second-order terms is also higher, $S$ $=0.4 \mathrm{D}, C=1.13 \mathrm{D}$ at $35 \mathrm{deg}$, than that obtained when considering the contributions of HOA: $S=0.08 \mathrm{D}, C=0.45 \mathrm{D}$ at $22 \mathrm{deg}$, but differences are now closer or even above $0.5 \mathrm{D}$ both for the sphere and cylinder.

\section{Discussion and Conclusions}

In summary, a theoretical framework to obtain the refractive error of an eye directly from aberrometric raw data was pre- sented. In the presence of sphere and cylinder refractive errors, beams are elliptical and vergences depend on orientation (meridian). That elliptical shape of lens power and beam vergences is described here by $2 \times 2$ symmetric matrices. The action of any combination of spherical and cylindrical lenses is described by $\mathbf{V}^{\prime}=\mathbf{P}+\mathbf{V}$, in matrix form, where the action of conventional lenses is represented as additive matrix operators. The matrix formalism becomes extremely useful to analyze the vergence error of a general skew ray from an aberrated beam. The vergence error of an aberrated ray is completely defined by the sum of two, one even-symmetric and one odd-symmetric $2 \times 2$ matrices. The odd (crosscomatic) vergence error cannot be corrected by standard lenses, but could be done by odd-symmetric lenses consisting of two positive and negative half-cylinders. Nevertheless, all odd-symmetric contributions to the global refraction are canceled by pupil average, and this cross-comatic vergence error is also canceled. Thus, that odd-symmetric matrix is irrelevant for the problem of refraction from aberrometry (but relevant for optical and image quality). The even-symmetric vergence error matrix defines an elliptical beam associated to the ray. The matrix formulation of both conventional refraction and of vergence error of an aberrated ray permitted one to apply the laws of standard clinical refraction to each ray passing through the pupil. This makes it possible to measure refractive error $(S, C$, and $\theta)$ for each ray and eventually correct it by the appropriated lenslet. The way to construct the vergence error matrix is straightforward from the aberrometric raw data, simply by computing the ratios (finite tangents) between image (centroids of spots) and pupil coordinates for each ray or aberrometric sample.

These results are a direct generalization of early aberrometry based on standard refraction. The difference was that 
Table 3 Example: Contributions of wave Zernike modes to the pupil average vergence error for subject $\mathrm{RN}, \mathrm{OD}, 2 \mathrm{R}=6 \mathrm{~mm}$.

\begin{tabular}{cccccc}
\hline$n$ & $m$ & $c_{n}^{m}(\mu \mathrm{m})$ & $\left\langle V_{\xi}^{\times}\right\rangle$ & $\left\langle V_{\eta}^{y}\right\rangle$ & $\left\langle V_{\eta}^{x}\right\rangle$ \\
\hline 2 & -2 & -0.1093 & 0 & 0 & -0.059 \\
2 & 0 & 0.1603 & 0.1234 & 0.1234 & 0 \\
2 & 2 & -0.4008 & -0.2182 & 0.2182 & 0 \\
4 & -4 & -0.0178 & 0 & 0 & -0.0125 \\
4 & -2 & -0.053 & 0 & 0 & -0.037 \\
4 & 0 & 0.3167 & 0 & 0 & 0 \\
4 & 2 & 0.0712 & -0.050 & 0.050 & 0 \\
4 & 4 & -0.0349 & 0.0245 & -0.0245 & 0 \\
6 & 0 & 0.0947 & 0.0643 & 0.0643 & 0 \\
\hline \multicolumn{7}{r}{ Second order only } & Total Vergence Error (diopters) & & -0.0595 \\
\hline
\end{tabular}

these measurements were mainly restricted to rotationally symmetric aberration modes, so that the vergence error was reduced to a single scalar number. To properly analyze today's aberrometric data, it is necessary to consider that vergence error of a skew ray is not a scalar anymore, but a $2 \times 2$ matrix.

A major issue is the possible singular points along both pupil axes. We have seen how the contributions of positive, $+\infty$, and negative, $-\infty$, singularities cancel by pupil average [see Eqs. (20)]. However, the singularities of vergence along the coordinate axes must be treated carefully when we have a discrete pupil sampling and, hence, a limited number of samples. A simple and direct solution is to take these data points out when computing the average. Ideally, one should design a sampling pattern avoiding both horizontal and vertical axes. If that is not possible, then the axial samples must not be considered for averaging [as formulated in Eq. (13)]. This would require having enough (off-axis) samples to compute the average. The computer simulation performed thus far showed that the quotients $X_{i, j} / \xi_{i, j}, Y_{i, j} / \eta_{i, j}$, etc., never gave singularities outside the axes, even for very high numbers of samples. A potential problem is the bias introduced by removing axial samples from the set used to compute the global vergence error. This step, namely, to pass from local (individual) refractive errors associated to each ray (or pupil sample), to a single global prescription, is crucial. Among the many possible choices, pupil integration, or average, presents many advantages, such as simplicity, robustness, an increase in signal-to-noise ratio, and being optimal under a linear leastsquares criterion. Nevertheless, the accuracy of this method appears to depend strongly on the total number of samples. For plain averaging, the number of samples needed to guarantee good accuracy is too high $(\sim 1000)$; Thus, practical implementations of the direct method would require more sophisticated integration and/or interpolation methods. Alterna- tively, one may use the present formulation to compute the refractive error from the wave aberration (Table 1). An important result is that this simple metric (pupil average) is consistent with well-known results when only second-order refractive errors are present (i.e., HOA-free wavefronts). Other robust criteria would give slightly different metrics (mode, etc.), or one could apply some pupil apodization weighting more (or less) different pupil zones. However, the direct way to obtain the prescription is to average the vergence error over the pupil.

The proposed direct method is opposite to the standard way in which one reconstructs the wavefront error and then attempts to predict clinical prescription by using image or visual quality metrics. Such indirect methods work by statistical correlations and may be good predictors of average tendencies, but may yield large errors for particular individuals. Instead of those image-quality metrics-based predictions, the use of the aberrometer as a refractometer instrument seems a better and more powerful strategy. Direct estimation of clinical refraction has been elusive until now, probably because of (not well-understood) interactions between higher and lower order wave aberration modes. The present formulation was used to study such interactions and to compute the contributions of all HOA Zernike modes to the average vergence error. Table 1 provides a way to compute vergence error from wave aberration, but this method is not necessary, if one can apply the direct way.

The present framework is different from previous approaches in two major aspects. On the one hand, there have been attempts to decompose vergence in terms of polynomial expansions, trying to mimic the treatment of wavefronts. ${ }^{7,8}$ For doing this, they typically consider only radial vergence, which is a single scalar magnitude. One can compute the radial vergence 


$$
\frac{\partial W}{\rho \partial \rho}=\frac{\xi}{\rho^{2}} X+\frac{\eta}{\rho^{2}} Y
$$

and then apply those data analysis schemes to obtain the refraction. The drawback of only using this scalar magnitude is that it involves the implicit assumption that the ray trajectory is going to always be coplanar with the $Z$-axis, which is not true for a general skew ray. On the other hand, the need of finding new basis functions to describe vergence is doubtful. Basis functions are useful in aberrometry to reconstruct the wavefront from the raw aberrometric data (partial derivatives), which requires a numerical integration, which is a noisy process. However, the vergence error for each aberrometric sample only involves the computation of coordinate ratios; the global refraction is obtained from these vergence errors by average. Eventually, one might use basis functions to perform interpolations or to improve numerical integration (average) over the pupil.

The framework presented thus far might have interesting implications in related fields, such as the analysis of corneal topographies, which deserve further developments. However, the main advantage of the present formulation is that it could help to move aberrometry closer to the clinical language and practice.

\section{Acknowledgements}

This research has been supported by the Spanish CICyT, Grants No. FIS2005-05020-C03-01 and No. FIS2008-00697. The author thanks Justo Arines for his help with Matlab programming.

\section{References}

1. L. N. Thibos, X. Hong, A. Bradley, and R. A. Applegate, "Accuracy and precision of objective refraction from wavefront aberrations," $J$. Vision 4, 329-351 (2004).

2. A. Guirao and D. R. Williams, "A method to predict refractive errors from wave aberration data," Optom. Vision Sci. 80, 36-42 (2003).
3. X. Cheng, A. Bradley, and L. N. Thibos, "Predicting subjective judgment of best focus with objective image quality metrics," J. Vision 4, 310-321 (2004).

4. R. A. Applegate, J. Marsack, and L. N. Thibos, "Metrics of retinal image quality predict visual performance in eyes with $20 / 17$ or better visual acuity," Optom. Vision Sci. 83, 635-640 (2006).

5. O. Nestares, R. Navarro, and B. Antona, "Bayesian model of Snellen visual acuity," J. Opt. Soc. Am. A 20, 1371-1381 (2003).

6. L. N. Thibos, W. Wheeler, and D. Horner, "Power vectors: an application of Fourier analysis to the description and statistical analysis of refractive error," Optom. Vision Sci. 74, 367-375 (1997).

7. D. R. Iskander, B. A. Davis, M. J. Collins, and R. Franklin, "Objective refraction from monochromatic wavefront aberrations via zernike power polynomials," Ophthalmic Physiol. Opt. 27, 245-255 (2007).

8. J. Nam, L. N. Thibos, and D. R. Iskander, "Zernike polynomials for analyzing refractive power maps derived from measurements of radial wavefront slope," Presented at 4TH European Mg. on Visual and Physiological Optics, Heraklion, Greece, 31 August-2 September 2008.

9. M. Koomen, R. Tousey, and R. Scolnik, "The spherical aberration of the eye," J. Opt. Soc. Am. 39, 370-376 (1949).

10. A. Ivanoff, "Les aberrations de l'oeil. Leur role dans l'accommodation," Éditions de la Revue d'Optique Théorique et Instrumentale, Paris (1953).

11. R. E. Bedford and G. Wyszecki, "Axial chromatic aberration of the human eye," J. Opt. Soc. Am. 47, 564-565 (1957).

12. W. N. Charman and J. A. M. Jennings, "Objective measurements of the longitudinal chromatic aberration of the human eye," Vision Res. 16, 999-1005 (1976).

13. H. C. Howland and B. Howland, "A subjective method for the measurement of the monochromatic aberrations of the eye," J. Opt. Soc. Am. 67, 1508-1518 (1977).

14. J. Liang, B. Grimm, S. Goelz, and J. F. Bille, "Objective measurement of wave aberrations of the human eye with the use of a Hartmann-Shack wave-front sensor," J. Opt. Soc. Am. A 11, 19491957 (1994).

15. R. Navarro and M. A. Losada, "Aberrations and relative efficiency of light pencils in the living human eye," Optom. Vision Sci. 74(7), 540-547 (1997).

16. R. Navarro and E. Moreno-Barriuso, "Laser ray tracing versus Hartmann-Shack sensor for measuring optical aberrations in the human eye," J. Opt. Soc. Am. A 17, 974-985 (2000).

17. M. Born and E. Wolf, Principles of Optics, 6th ed., Pergamon Press, Oxford (1993). 